Utilization of Magnetorheological Finishing as a Diagnostic Tool for Investigating the Three-Dimensional Structure of Fractures in Fused Silica

J. A. Menapace, P. J. Davis, W. A. Steele, L. L. Wong, T. I. Suratwala, P. E. Miller

November 15, 2005

Boulder Damage Symposium XXXII

Boulder, CO, United States

September 19, 2005 through September 21, 2005 
This document was prepared as an account of work sponsored by an agency of the United States Government. Neither the United States Government nor the University of California nor any of their employees, makes any warranty, express or implied, or assumes any legal liability or responsibility for the accuracy, completeness, or usefulness of any information, apparatus, product, or process disclosed, or represents that its use would not infringe privately owned rights. Reference herein to any specific commercial product, process, or service by trade name, trademark, manufacturer, or otherwise, does not necessarily constitute or imply its endorsement, recommendation, or favoring by the United States Government or the University of California. The views and opinions of authors expressed herein do not necessarily state or reflect those of the United States Government or the University of California, and shall not be used for advertising or product endorsement purposes. 


\title{
Utilization of Magnetorheological Finishing as a Diagnostic Tool for Investigating the Three-Dimensional Structure of Fractures in Fused Silica
}

\author{
Joseph A. Menapace*, Pete J. Davis, William A. Steele, Lana L. Wong, \\ Tayyab I. Suratwala, and Philip E. Miller \\ University of California, Lawrence Livermore National Laboratory \\ P.O. Box 808, 7000 East Avenue, L-491, Livermore, CA 94550 \\ 925-423-0829 (menapace1@1lnl.gov)
}

\begin{abstract}
Work performed under the auspices of the US Department of Energy by Lawrence Livermore National Laboratory under contract No. W-7405-ENG-48 with the LDRD program.
\end{abstract}

\begin{abstract}
We have developed an experimental technique that combines magnetorheological finishing (MRF) and microscopy to examine fractures and/or artifacts in optical materials. The technique can be readily used to provide access to, and interrogation of, a selected segment of a fracture or object that extends beneath the surface. Depth slicing, or cross-sectioning at selected intervals, further allows the observation and measurement of the three-dimensional nature of the sites and the generation of volumetric representations that can be used to quantify shape and depth, and to understand how they were created, how they interact with surrounding material, and how they may be eliminated or mitigated.
\end{abstract}

Keywords: subsurface damage, MRF, brittle fracture, surface imperfections, laser-induced damage

\section{INTRODUCTION}

A major attribute of optical fabrication is connected with the management and control of surface or near-surface imperfections and fractures ${ }^{1-6}$. Incorrect data or awareness on these kinds of artifacts typically leads to lower quality products and a constant struggle with high rework rates. To gain an understanding of process-induced fractures and imperfections on optical surfaces, a practical approach is needed that yields sufficient detail and information about these artifacts. The method not only needs to yield information about the surface characteristics of an optic at a particular point in the fabrication process, it needs to also give information on how fractures and imperfections vary from the surface into the bulk material. Traditional methods are difficult to use when studying fractures in optical surfaces for a number of reasons. Pad or lap polishing, in addition to removing material at a low rate, tends to move material about an optical surface due to chemo-mechanical processes. This results in re-deposition of modified material (usually weakly-structured hydrated material) onto the surface that masks or buries surface fractures ${ }^{7}$. Even though undetectable due to a filling in with closely index-matched material, these fractures ultimately limit the performance of the optic under high stress conditions caused by high pressure, large thermal gradients, and intense laser light. Traditional polishing is also difficult to control and gauge when being applied to remove a small specified amount of material and is prone to imparting its own damage due to contributors such as slurry and pad (lap) agglomeration, contamination, communition, etc.

Over the past few years we have realized success in using magnetorheological finishing (MRF) to polish optics possessing little to no surface or subsurface fractures with a goal of improving their high fluence ultraviolet laser damage resistance ${ }^{8}$. The MRF technique is deterministic making it easily controllable in removing a prescribed amount of material from an optical surface. Moreover, it is reproducible and can be applied 
repeatedly to a high degree of precision with depth and form control to better than $20 \mathrm{~nm}^{9,10}$. MRF polishing has also been shown to expose and remove fractures without adding damage. Expanding on this concept, we have developed a technique that utilizes MRF as a diagnostic tool to characterize fractures and imperfections on and below optical surfaces. This paper describes the three-dimensional MRF technique and presents example experiments performed on fused silica optics illustrating how it can be used to study fractures.

\section{MRF THREE-DIMENSIONAL ANALYSIS PROCESS}

The MRF three-dimensional analysis process incorporates three systematic steps to interrogate the detailed morphology of individual fractures or fracture networks in optical materials. These steps include: 1) sample preparation, 2) MRF cross-sectioning and 3) analysis of the area of interest (AOI) as depicted in Figure 1. Sample preparation is typically conducted only once during the process with MRF cross-sectioning and AOI analysis performed iteratively until adequate data is collected to assemble a morphological rendering (usually complete penetration and removal).

For step 1, optical samples being prepared for interrogation can be obtained in a variety of ways. They can be drawn from a specific step of a manufacturing process, such as a particular grinding or polishing operation. These are what we call process-related specimens. Process-related specimens are typically obtained by processing actual work pieces, by using surrogate samples processed in the same manner as production pieces, or they can be cut from actual production work pieces if one is interested in studying processes used on large (for example $0.5 \mathrm{~m}$ ) aperture optics.

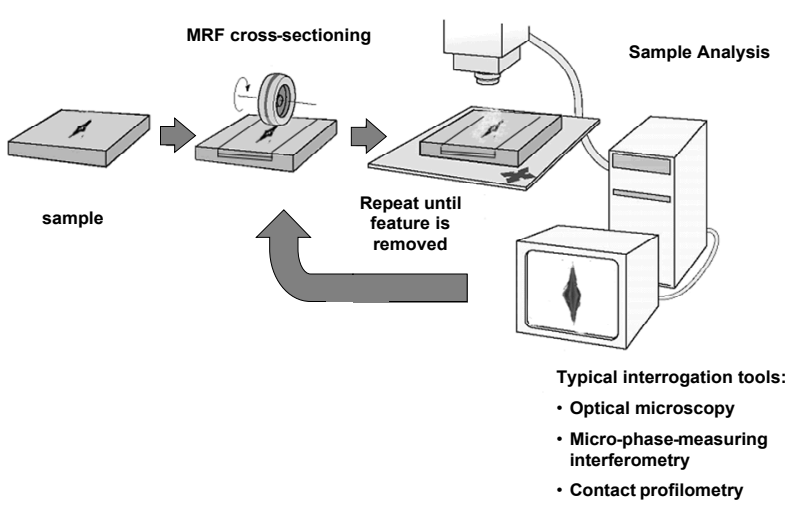

Figure 1: The three-dimensional MRF analysis process involves three major steps - 1) sample preparation, 2) cross-sectioning, and 3) sample analysis. Steps 2 and 3 are iteratively performed until the process is complete.
Process-related specimens collected from polishing operations typically contain isolated fractures, scratches, or micro-scratches ("sleeks") arising from process contributors such as outlier or ill-defined abrasive size distributions, or abrasive/lap contamination ${ }^{11}$. They could also contain artifacts from cleaning and handling and fracture networks resulting from insufficient material removal in previous grinding steps. In general, process-related specimens obtained from grinding operations contain surface morphologies consisting of fracture networks from the most recent grinding step $^{12}$. Remnants from previous grinding operations may be present depending upon whether or not sufficient material was removed during subsequent process steps. During our experiments, we have found that fracture networks from grinding and isolated imperfections from polishing can be prepared for examination. This is accomplished by either adequate material removal during the process under study or by starting with a polished and well-examined specimen. In the latter case, fractures or imperfections from the last process step are the only ones present or are the dominant fracture network in the surface. An example of the three-dimensional MRF analysis obtained from a process-related specimen made using a 120-grit fixed abrasive grinding process is discussed later in this manuscript. This specimen was prepared from a polished fused silica substrate by grinding on a Blanchard Model 11A20 using a 120 grit (125 micron) diamond in a metal matrix tool (downward feed rate $=250 \mathrm{microns} / \mathrm{min}$, rotation rate $=45 \mathrm{rpm}$, time $=20 \mathrm{sec})$.

Test sample are also prepared by applying imperfections to the polished optical surface using standard indentation tools, or by dragging abrasives or diamond tipped tools across the surface. These imperfections are what we call engineered imperfections. These imperfections generally include single fracture sites, isolated trailing fracture networks, micro-fractures (sleeks), or densified (compacted) material zones arising from plastic movement of material on, or near, the optical surface. In our laboratory, we have been successful in applying engineered imperfections to fused silica using a Shimadzu HMV-2 micro-hardness tester. Knoop, Vickers, and 
Brinell indentation tool tips are readily available for this instrument and can be easily used to make indentations and fractures in optic surfaces under different load conditions ${ }^{13}$. The morphology of an engineered imperfection made using a Knoop indenter under a $4.9 \mathrm{~N}$ applied load is discussed later in this manuscript as an example of the usefulness of the three-dimensional MRF process in studying the characteristics of surface imperfections.

Wet etching via an aqueous acid or base solution develops the specimens for subsequent processing and characterization. Hydrofluoric acid etching of fused silica can be used to adequately and reproducibly open fractures present at the optical surface that are closed or optically contacted to neighboring material and difficult to observe during microscopic examination ${ }^{8,11,12}$. It also exposes subsurface damage generated during the fabrication process that has been subsequently buried under a re-deposited layer of refractive index matched hydrated-glass. A fifteen minute etch using a 20:1 ammonium fluoride/ hydrofluoric acid solution (commercially known as 20:1 buffered oxide etch) adequately develops the surfaces by opening fractures to a width of about 0.8 microns with about 0.4 microns of material removal from the optic surface.

Once the samples are prepared, MRF cross-sectioning, Step 2, is used to remove plane-parallel sections of predetermined thickness from the specimen surface (Figure 2). Material removal is accomplished by rastering the surface using a well-characterized MRF removal function at uniform velocity. The MRF software is used to set the removal depth using a convergence routine with a flat starting and ending topography. In our experiments, a $10 \times 10 \mathrm{~mm}$ area was selected for raster cross-sectioning to balance polishing time and available analysis area. Raster cross-sectioning times averaged about 3 minutes per cycle. We obtained cross-section depths as low at $100 \mathrm{~nm}$ and as high as $600 \mathrm{~nm}$ per cycle depending upon the MRF volumetric removal. An analysis sequence, Step 3, follows each MRF raster cycle. MRF crosssectioning and subsequent analysis continues until the imperfections are removed. Material removal is monitored using a KLA Tencor P-10 surface profilometer after each cycle. This is done by measuring the surface profile with line scans that extend over both the sectioned and surrounding areas on the optic and computing the height difference between the sectioned and un-sectioned areas. The

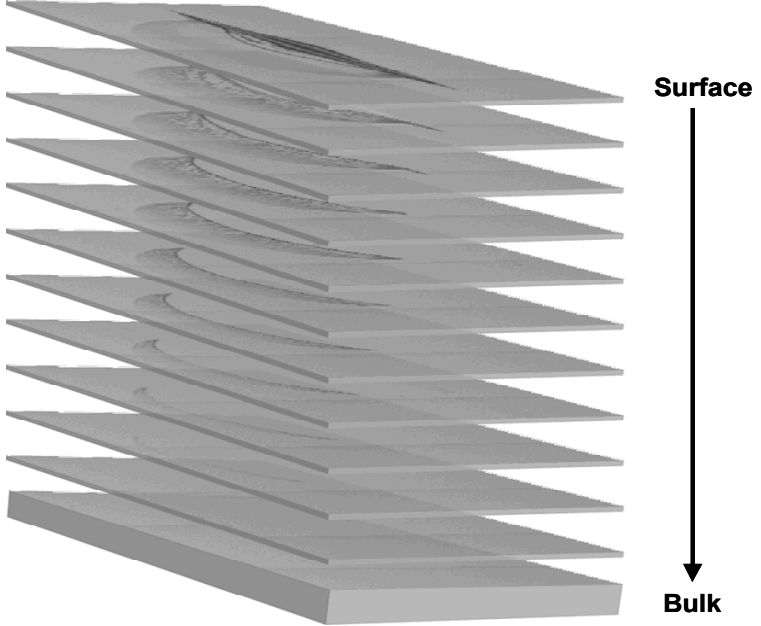

Figure 2: MRF cross-sectioning uniformly removes material from the AOI yielding depth-sliced information at prescribed intervals from the surface into the bulk. accuracy of this measurement is about $+/-5.0$ angstroms.

We utilized three different techniques to analyze the defects being cross-sectioned, step 3. In general, any combination of nondestructive analysis techniques can be utilized for this portion of the process. The techniques we make use of are based upon the type of observables we are looking to obtain data on and upon the availability of the instruments for our work. First, a Nikon NEXIV VMR series CNC based optical measuring system is used to image each polished cross-section. Micrographs obtained using the NEXIV system are typically collected using episcopic lighting at 300x magnification to resolve detail. One major advantage of using this computer-based measuring system is its ability to perform repeated tasks at high speed. Once the defect locations are registered in the system, the analysis can be automated. This is particularly useful in analyzing engineered imperfections since they are typically spaced far enough away from neighboring imperfections to avoid any influence during their preparation. The computer system can be programmed to allow the microscope stage to jump between several areas on the optical surface and perform analyses on several defects during a single process step without wasting time scanning over surrounding areas. The NEXIV system has laser and contrast auto-focusing capability which maintains a clear image of the surface as the crosssectioning proceeds. Defect areas covering more than one field-of-view are acquired in series using the instrument's computer controlled stage that is programmed to collect tiled imagery. Tiled images with five percent overlap were stitched together using Adobe Photoshop ${ }^{\mathbb{B}}$. Three-dimensional renderings of the imperfections are generated using the entire set of cross-sections collected and software written by the primary author to place the imagery in its appropriate location in all three dimensions. Briefly, this software overlays the 
images at the correct space coordinates and uses image alpha transparency manipulation (magnitude and threshold) to generate surface topography. The software also allows the user to rotate and translate the renderings in three-dimensions thereby offering different views of the defect structure. Layers composing a rendering can be made invisible to expose information from the remaining layers (interior) of the imperfection. We also use a Wyko NT200 white light micro-phase-measuring interferometer to investigate the imperfection surface topography and area surface finish during the sectioning process. This technique allows measurement of small changes in surface topography as well as identification and characterization of zones within the AOI that possess different optical characteristics such as refractive index or density. Lastly, in addition to monitoring the progress of the cross-sectioning, a KLA Tencor P-10 surface profilometer is used to measure the surface topography and roughness. This technique involves contact on the optic surface and provides for independent data on the surface structure and finish of imperfections. Data collected using the contact profilometer is compared data obtained from white-light interferometry or used an alternative on surfaces where interferometry fails (ground glass, steep gradients, or deep fractures).

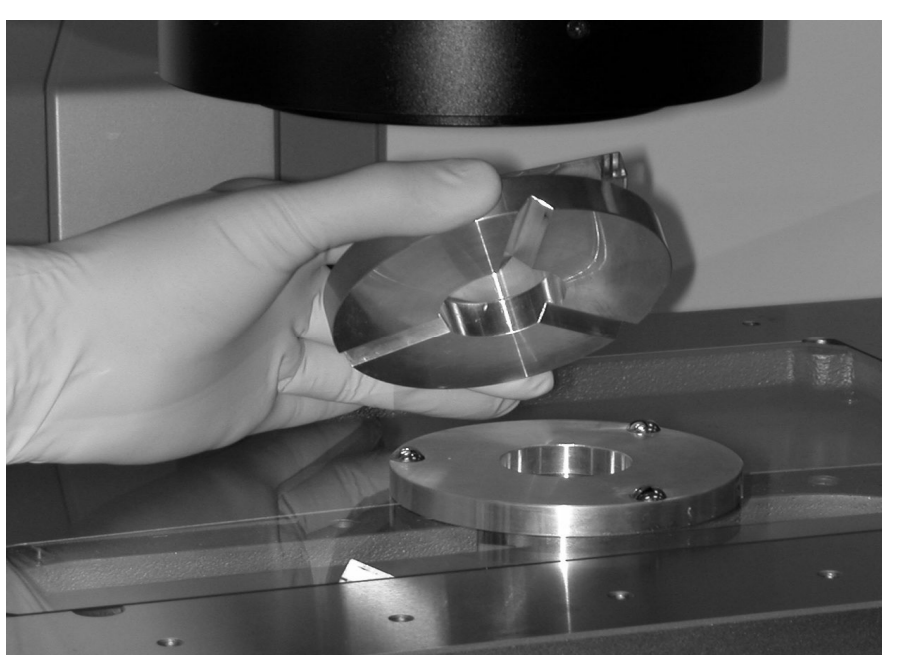

Figure 3: A kinematic sample holder is used to accurately and repeatedly place optics onto the various tools used for AOI analysis.

To provide repeatability and processing efficiency during MRF cross-sectioning and sample analysis, we designed and fabricated a kinematic sample holder that could be attached to the optic to facilitate placement onto the various instruments used for the process (Figure 3). The sample holder is comprised of two doughnut-shaped aluminum plates one of which has radial grooves spaced 120 degrees apart cut into its lower surface. The corresponding mating plate has stainless steel ball bearings placed at 120 degrees along a circle centered on the plate. The grooved plate and the optic being studied are glued together using vinyl polysiloxane dental impression epoxy (Examix NDS, GC America, Inc.). The mating plate is attached to the analytic instrument used for interrogation of the optic. Several mating plates can be used if the analysis involves more than one instrument or technique. When these plates are placed together during use, accurate and reproducible placement of an optic is attained to less than 2 microns. Accurate and reproducible X$\mathrm{Y}$ placement of the optic is critical to this technique as its goal is to unravel the spatial relationship of imperfections in the AOI as it is cross-sectioned in $\mathrm{Z}$ resulting in a rendering of its three-dimensional character. Both plates have an opening in the center so that transmission experiments or diascopic lighting techniques can be employed. The opening in the center of the grooved plate also allows the optic to remain in the sample holder during MRF-cross sectioning. Vacuum chucks used to hold the optic on the MRF can be inserted into this opening and used to engage the optic onto the spindle during material removal.

\section{THREE-DIMENSIONAL MRF ANALYSIS OF PROCESS-RELATED SPECIMENS}

One of the important aspects of optical fabrication above and beyond figure control is the understanding of process-induced fractures in optical surfaces, particularly if one is interested in making laser damage-free parts. Process-induced fractures dictate how much material needs to removed during subsequent steps ${ }^{11,12,14}$. They also establish what processes will need to be used to yield an optic possessing a superior surface finish. Lack of knowledge or an insufficient understanding of process-related fractures all too often leads to insufficient material removal during intermediate fabrication steps. This can result in presence of fractures and imperfections in the final optic, can cause the optic to have to be taken backwards in the process for rework, or can add time to subsequent fabrication steps, particularly polishing, that reduces productivity and increases fabrication costs. In other work, we have developed techniques to measure the depth profile of surface fractures and sub-surface damage on optical surfaces during various portions of the fabrication process ${ }^{14}$ and have gained an understanding of the mechanics involved in the formation of fractures during optical fabrication. Three- 
dimensional analysis of the fracture networks present during optic fabrication adds to one's understanding and interpretation.

Figures 4-6 present the results of the three-dimensional MRF analysis applied to a piece of fused silica ground using a 120-grit $(125 \mu \mathrm{m})$ fixed abrasive and then etched. The micrographs in Figure 4 show the cross-sections taken during the process from the fused silica surface to 75.1 microns into the bulk material in an average of 3.2 micron increments. The micrograph collected at the surface dense fracture network and is very difficult, if not impossible, to interpret due to the significant amount of residual rubble present. This image represents what is

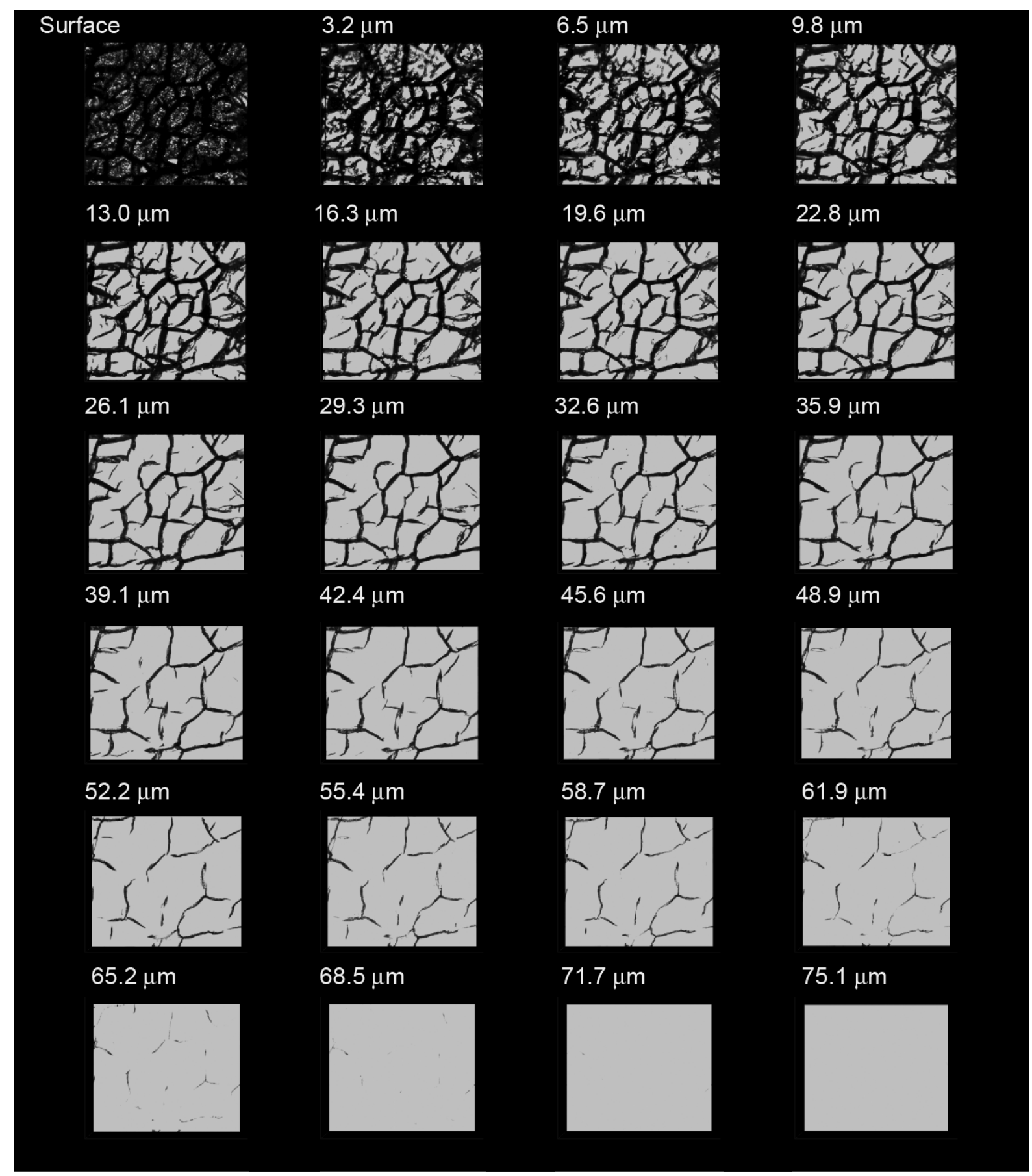

Figure 4: Micrographs taken during MRF cross-sectioning of a fused silica specimen that had been ground using a 120 grit fixed abrasive grinding process. High-magnification images from each layer are stitched together yielding large-field, highresolution two-dimensional views. Characteristic fracture patterns and fracture lengths are observed peculiar to this process's parameters and material removal characteristics. 
seen in a microscope if one is trying to assess fractures or damage on a ground glass surface. Cross-sectioning just a few microns into the surface using the MRF, however, starts to reveal the details of the fracture network arising from this particular grinding process. The rubble zone extends about 6 microns into the bulk and a marked decrease in shallow fractures is observed at 16 microns into the bulk. Beyond this point, most of the heavy fracture network is penetrated and the characteristic fracture network for this process dominates. Characteristic fracture patterns and fracture lengths are observed that are peculiar to this process's type and material removal conditions which represent signatures for this process step during optical fabrication. Here, the fracture network which is composed of concatenated fractures of a specific length breaks up into radial fractures possessing the characteristic length. This pattern continues with no or little change in crack length until the network is entirely removed at 75 microns into the bulk.

Figure 5 illustrates the three-dimensional reconstruction of the MRF cross sections. The reconstructed image highlights the fracture networks as observed from the exterior of the optic if one could discern the crack network depths to some extent into the surface. In this view, the dominant fracture network in the optic surface

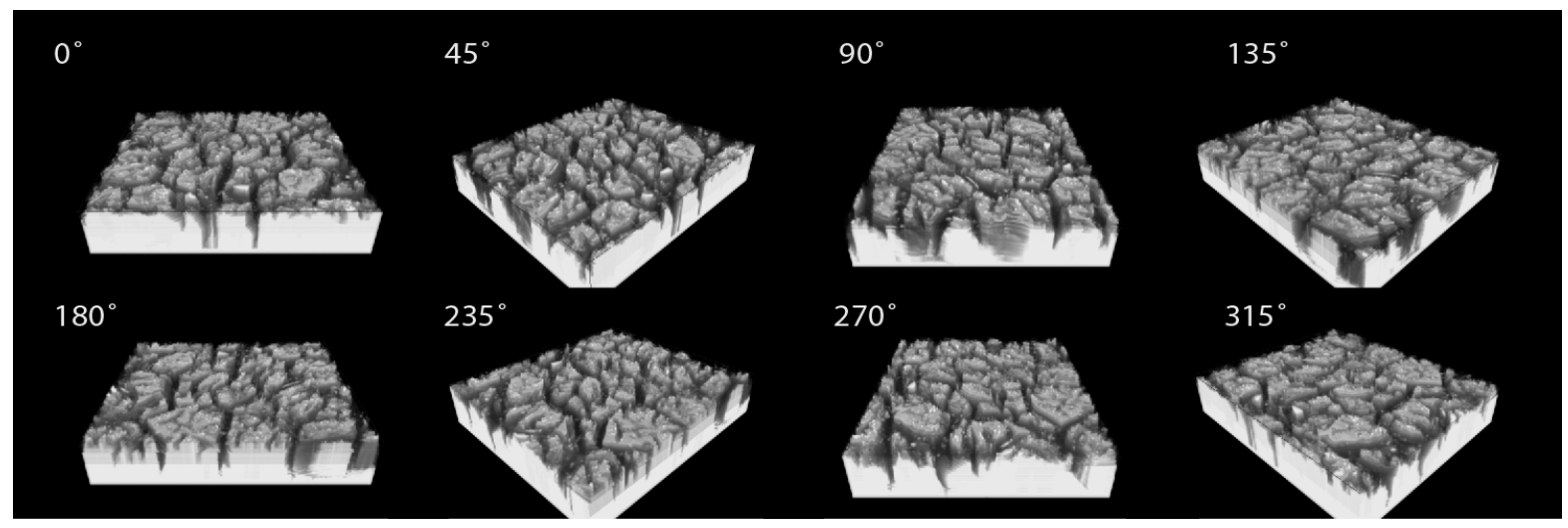

Figure 5: Three-dimensional reconstruction of the MRF cross-sections for the 120 grit ground specimen shows the fracture network as observed from the exterior of the optic similar to that observed via edge polishing.

is quite visible, revealing process dependent structure. It is composed of concatenated radial fractures yielding a mosaic appearance to the reconstruction. In the rotated views, the radial crack network along the rendering's edges gives some indication of the orientation and extent of the deep fractures relative to the surface. This is equivalent to what is observed after edge polishing. The three-dimensional reconstruction can also be manipulated to yield a topographical image of the fracture network interior which is equivalent to looking at the network from the bulk region, Figure 6. These views reveal the fracture morphology and the concatenation of the small radial cracks into large crack networks. They also indicate that the dominant radial fractures have a high aspect ratio and penetrate into the surface to about the same depth before they rapidly terminate. In other

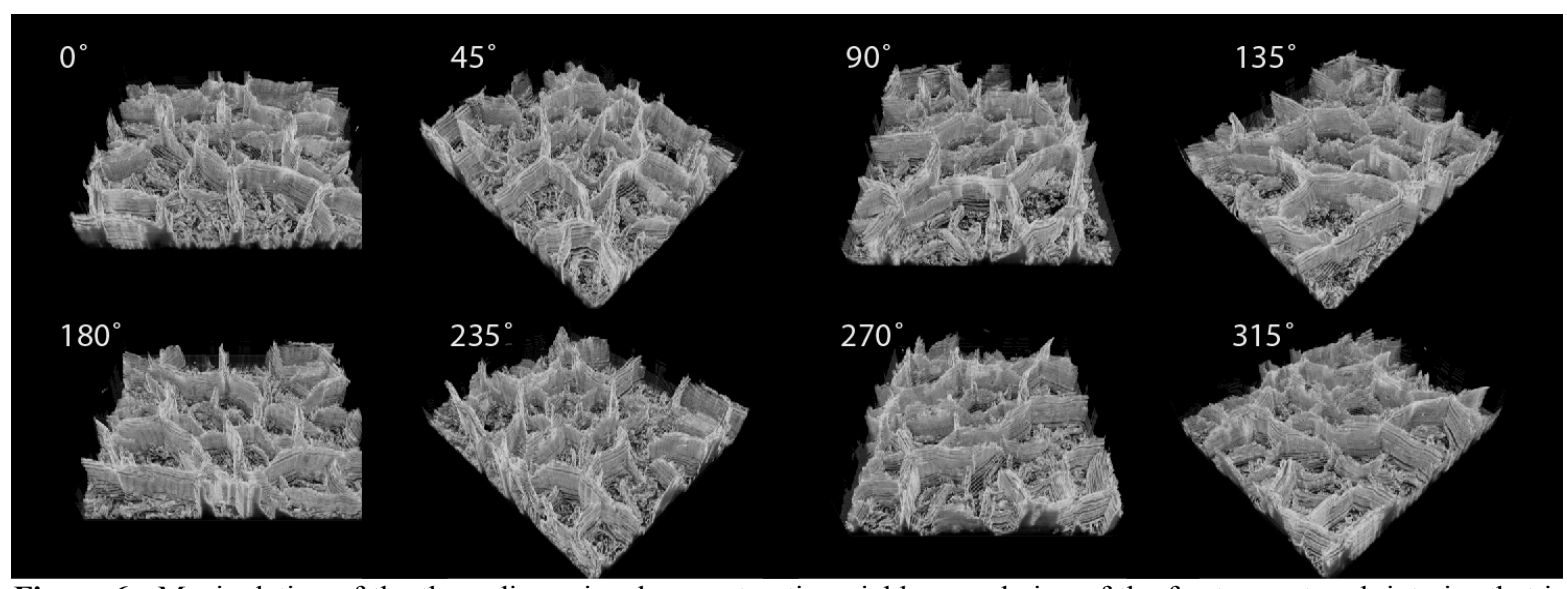

Figure 6: Manipulation of the three-dimensional reconstruction yields a rendering of the fracture network interior that is equivalent to looking at the network from the bulk region. These views reveal the fracture morphology and the concatenation of small radial cracks into large crack networks. 
words, the radial fractures can be represented by high-aspect rounded rectangles openings into the surface. They also only possess slight curvature away from normal. Further interpretation of the views shown in Figure 6 reveals that the shallower fractures look much like the deeper fractures signifying that they are from the same process but occurred earlier in time. They are shallower in appearance due to material removal occurring in the grinding process. This suggests that the deepest fractures are those that occur late in the grinding process, perhaps during the last few rotations of the grinding segments or grinding passes. Shallower damage most likely started out as deep fractures but is shortened as the grinding process proceeds. This also implies a well-known fact that lateral cracking is responsible for material removal and that radial fractures are associated with surface or subsurface damage.

\section{THREE-DIMENSIONAL MRF ANALYSIS OF ENGINEERED IMPERFECTIONS}

The study of isolated fractures and imperfections in optical materials can be used as a means to understand the details and mechanisms involved in the deformation process that ultimately leads to plastic flow and fracture in brittle materials. The majority of the work done in this area involves interrogating the surface imperfection's morphology using a microscope and interpreting its subsurface behavior based upon what is occurring at the surface. Having the ability to observe what is occurring in the material as one moves from the surface is crucial to verifying assumptions made about the material's behavior beneath the surface and to reveal characteristics that may be masked by activity at the surface. As an example on how the three-dimensional MRF analysis technique can be applied to engineered imperfections, we present the results obtained from a Knoop indentation applied to the surface of polished fused silica.

The Knoop indenter ${ }^{13}$ has a prescribed geometry designed to impart asymmetric stresses in a test sample. It is composed of a rhombohedral-shaped diamond cut to have 130 degree and 172.5 degree included angles between its facet diagonals. This results in an ideal diagonal length ratio of 7.114 between the long and short diagonals of an indentation applied normal to the place of an optic. Under ideal conditions, the Knoop indenter penetration depth, $\mathrm{D}$, should therefore be $\mathrm{D}=\mathrm{H} / 2 \tan (3.75 \mathrm{deg})=\mathrm{W} / 2 \tan (25 \mathrm{deg})$ depending upon whether

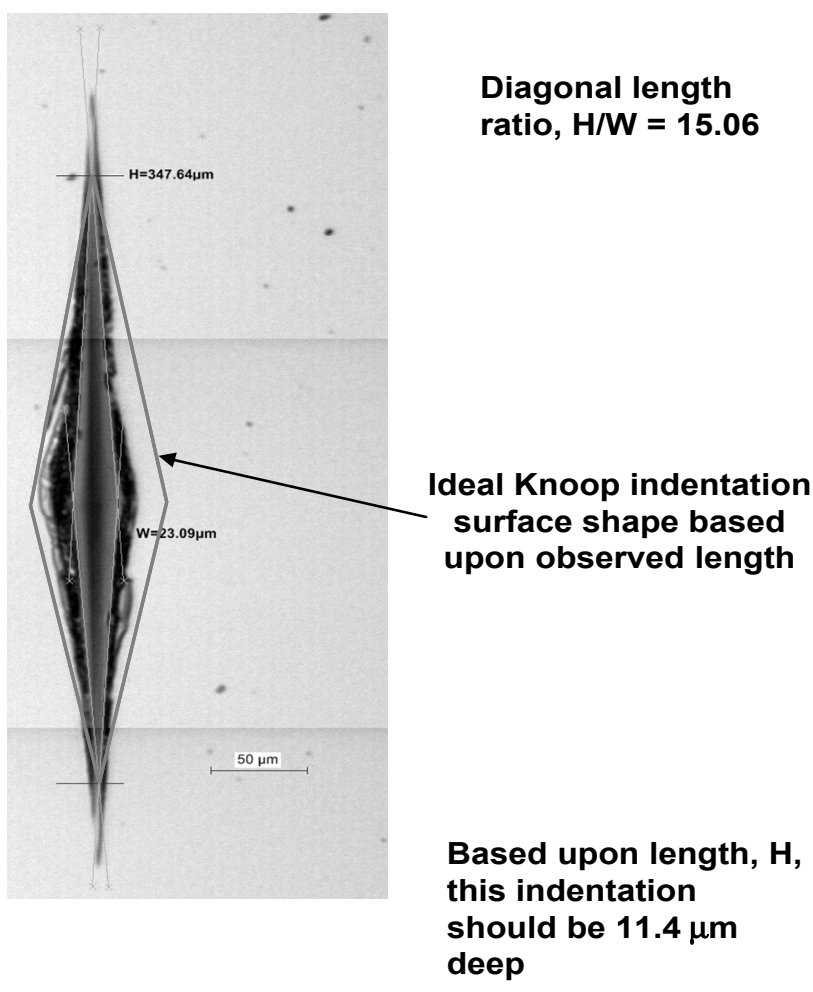

Figure 7: Micrographs of Knoop indentations in fused silica show a non-ideal aspect ratio indicating the effects of elastic recovery. one measures the length of the long, $\mathrm{H}$, or short, $\mathrm{W}$, diagonal.

Figure 7 presents a micrograph of a Knoop indentations applied to fused silica. The diagonal length ratio measured at the surface is 15.06 , over a factor of two greater than the indenter diagonal length ratio indicating that non-perfect behavior occurred during the indentation process. This behavior points to elastic recovery/deformation. Moreover, based upon the long diagonal length of 347.64 microns, the indentation should be 11.4 microns deep. Considering the non-ideal behavior of the indentation, one can estimate the amount of elastic recovery that occurred after the load on the indenter is removed by looking at the short diagonal length, 23.09 microns, compared to its ideal value of 48.87 microns. Parallel to the fused silica surface, 53 percent elastic recovery occurs after the Knoop indenter load is removed. Based upon the difference between the measured and ideal indentation short diagonal lengths, the elastic recovery occurring normal to the facet faces should be 5.45 microns which estimates the amount of elastic recovery from the bottom of the indentation to be 6 microns. 
Figure 8 shows the results of MRF cross-sectioning performed on the indentation. Complete removal of the physical indention occurs between 1.8 and 2.4 microns into the bulk fused silica. The indentation's footprint continues to a depth between 6.0 and 6.6 microns. Even though the prediction on the elastic recovery from the surface measurements compares well to that measured, the true recovery is larger. A compacted zone of modified fused silica exists underneath the physical indentation between 2 and 6 microns. Including the compacted/modified fused silica zone, 82 percent total indentation recovery occurs normal to the optical surface

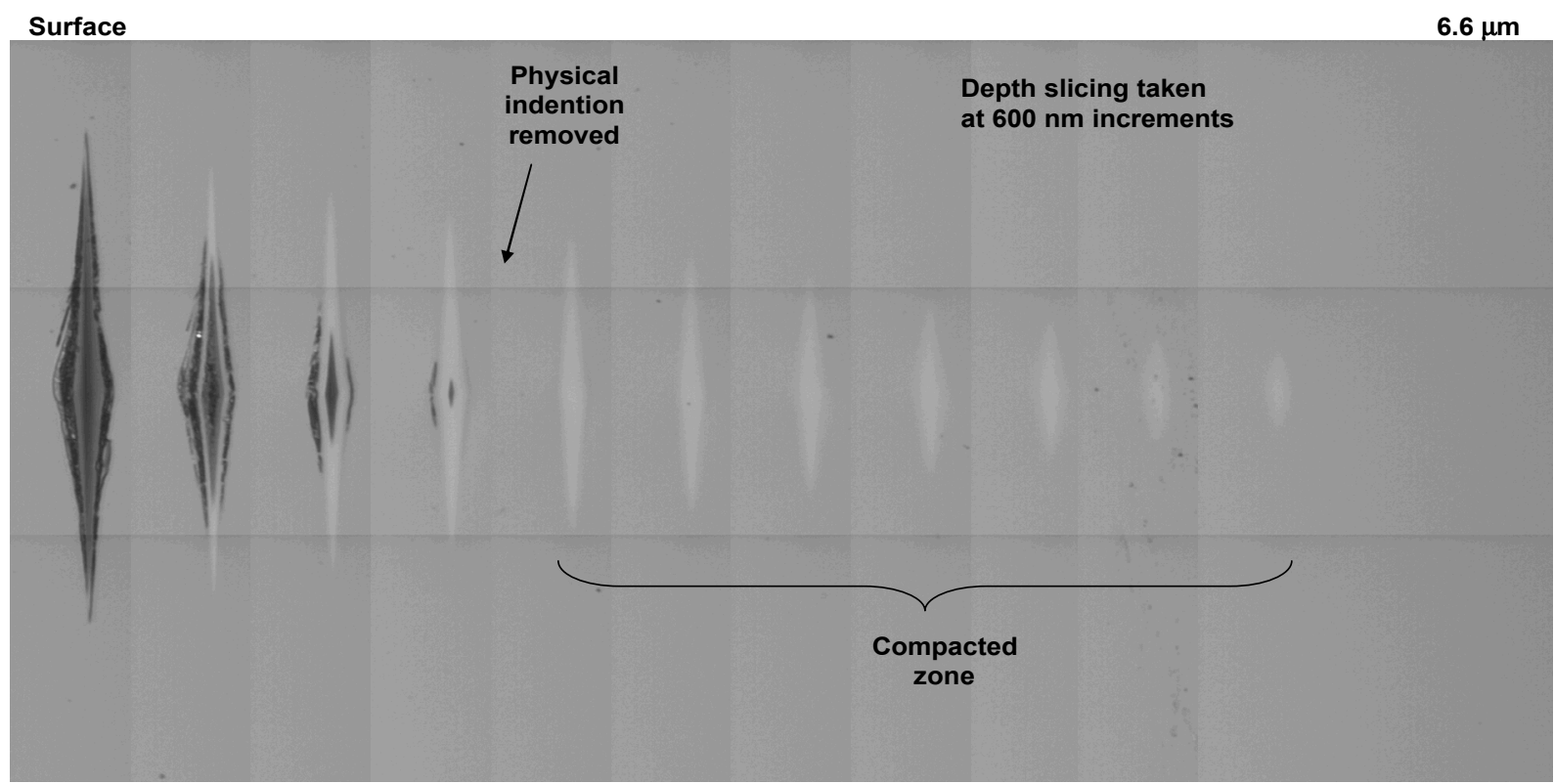

Figure 8: Optical Micrographs taken during MRF cross-sectioning reveal the characteristics of the Knoop indentation from the surface to the interior of the fused silica sample. Complete removal of the physical indentation occurs between 1.8-2.4 microns. The indentation's footprint that is comprised of a compacted zone continues to a depth between 6.0 and 6.6 microns.

which is composed of 37 percent modified fused silica and 45 percent elastic recovery from normal fused silica ${ }^{15}$. The existence of the compacted fused silica zone can be rationalized by the extreme pressures present at the indentation tip during the loading process. From the geometry of the Knoop indenter and the applied load of $4.9 \mathrm{~N}$ used, the compressive stress at the indenter/fused silica contact falls below $2 \mathrm{GPa}$ beyond 5.5 microns in depth. This is the point where elastic formation begins and the indenter tip continues to penetrate without causing permanent deformation. At lower penetration depths during the loading process, compressive stresses as high as $14 \mathrm{GPa}$ are present at 2 microns into the surface. These stresses are above the compressive yield strength of fused silica and result in compaction or modification (densification). Figure 9 shows a three-dimensional rendering of another Knoop indentation containing both a compacted fused silica zone and a large hoop fracture running parallel to the long diagonal axis. Its existence suggests that the compacted fused silica zone is formed during compression (loading). It also suggests that the fracture is formed during the recovery (unloading) process where tensile stresses dominate.

The compacted fused silica zone has a different refractive index and density than the surrounding fused silica hence it is observable in the microscope imagery and in the white light interferometry. During initial analyses on Knoop indentations made at low load $(0.2 \mathrm{~N})$ using the white light interferometer,

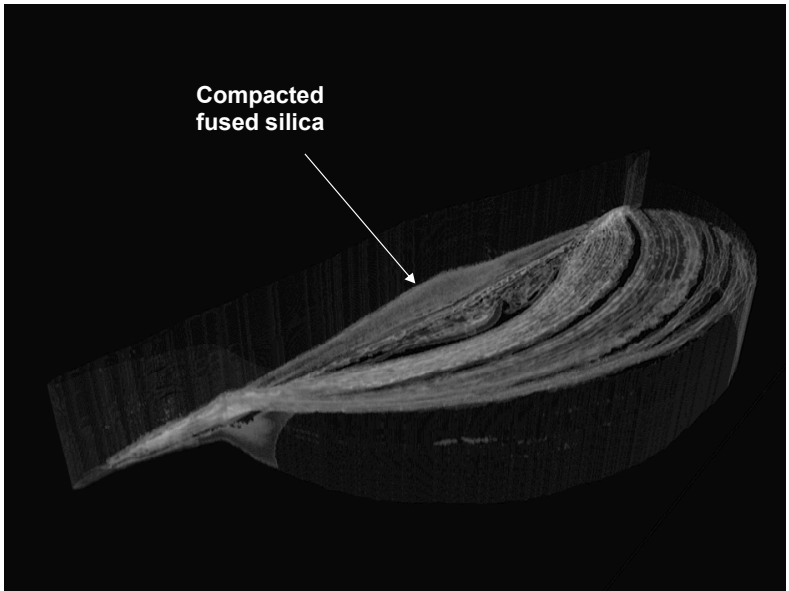

Figure 9: Three-dimensional reconstruction of a Knoop indentation showing the details of the indentation and its associated compacted zone and hoop fracture. 
measurements of the cross-sections suggested that the compacted layer is very shallow, approximately $4 \mathrm{~nm}$, and should be removed with the next MRF cross-section pass (Figure 10). After several further cross-sections,
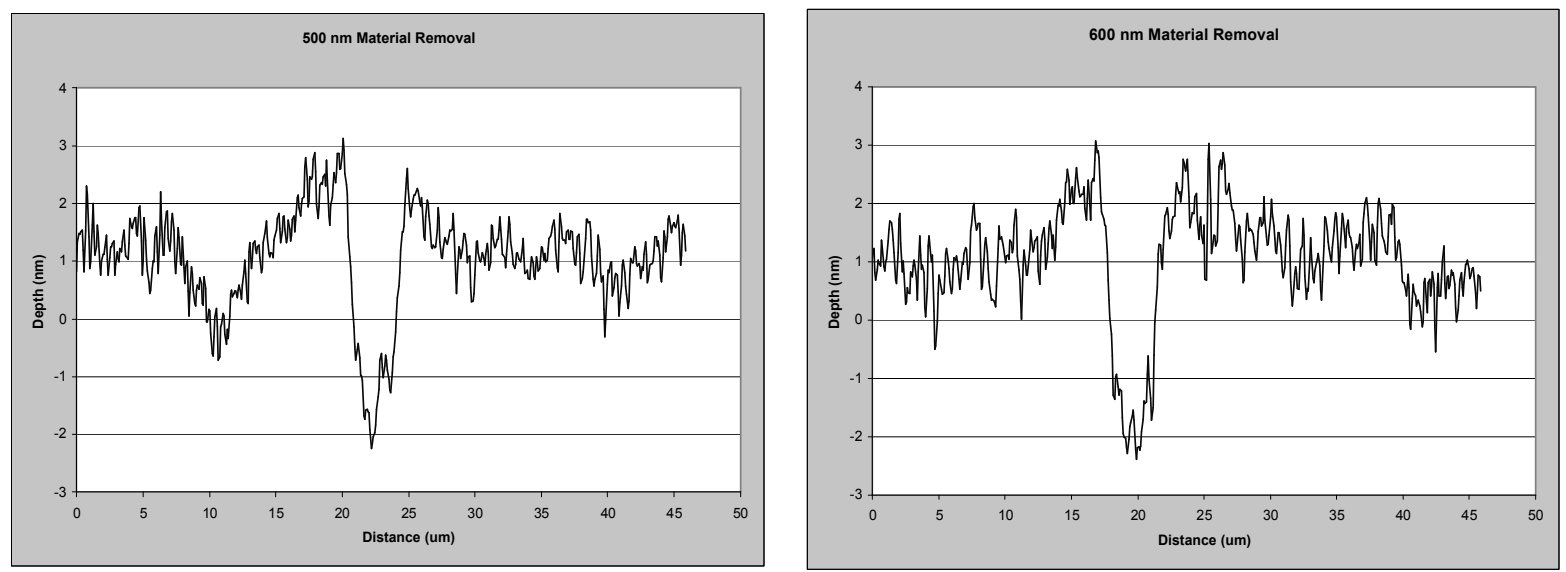

Figure 10: White light interferometry measurements of a Knoop indentation cross-section after $500 \mathrm{~nm}$ material removal (left) indicates that the compacted layer is very shallow and should be removed with the next cross-section removal pass. The apparent depth remains at about $4 \mathrm{~nm}$ after $600 \mathrm{~nm}$ material removal. This apparent depth remained nearly constant until the compacted zone was removed $(1750 \mathrm{~nm})$ suggesting that the apparent depth is an artifact of the measurement.

the apparent feature depth remains nearly constant indicating that the measurement is not real. Measurements conducted on the AOI using the stylus contact profilometer indicate that the surface did not contain an indention. The surface in these measurements only contains 5-7 angstroms (rms) micro-roughness representative of the specimen's overall surface character. It is therefore an artifact arising from the different refractive index of the compacted layer which alters the phase of the reflected light from the surface similar to

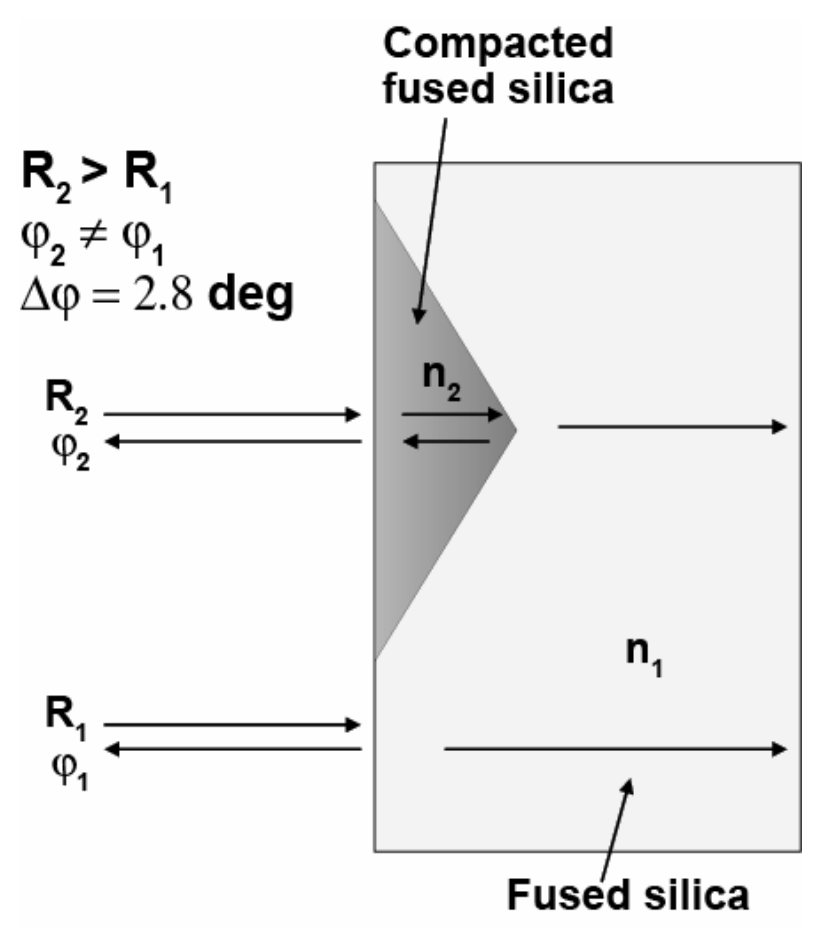

Figure 11: The refractive index for the compacted layer can be estimated using the phase change observed and a model implementing a thin layer of material containing a modified refractive index over a semi-infinite backing of normal fused silica. that occurring in thin film coatings, Figure 11. The refractive index for the compacted layer can be estimated using the phase change observed and a model implementing a thin layer of refractive index, $\mathrm{n}_{2}$, over a semi-infinite backing ${ }^{16-18}$ containing normal fused silica, $\mathrm{n}_{1}=1.457$.

1) $\tan \bar{\varphi} \cong \frac{\left[\left(\left(n_{1}+1\right)\left(\frac{n_{1}}{n_{2}}-n_{2}\right)+\left(n_{1}-1\right)\left(\frac{n_{1}}{n_{2}}+n_{2}\right)\right)\right]}{\left[\left(n_{1}-1\right)\left(n_{1}+1\right)-\left(\frac{n_{1}}{n_{2}}-n_{2}\right)\left(\frac{n_{1}}{n_{2}}+n_{2}\right)\right]}$

In deriving Equation 1, the phase terms for propagating and reflecting wavefronts are averaged to account for the dispersion of light in the interferometer and the varying thickness of the compacted layer leading to an average observed phase for the returning light. This phase is recorded as a difference in phase from 180 degrees. Using this relationship the refractive index for the compacted fused silica layer is estimated to be 1.486 .

The refractive index for the compacted layer can also be estimated using the intensity difference observed 
using episcopic microscopy and the Fresnel relation:

2) $n_{2}=\frac{\left(\frac{I_{r s}}{I_{r i}}\right)^{\frac{1}{2}}\left(\frac{n_{1}+1}{n_{1}-1}\right)+1}{\left(\frac{I_{r s}}{I_{r i}}\right)^{\frac{1}{2}}\left(\frac{n_{1}+1}{n_{1}-1}\right)-1}$

where $I_{r i}$ is the intensity of the reflected light from the compacted layer and $I_{r s}$ is the intensity of light from the surrounding fused silica. In this case, a refractive index of 1.481 is obtained. These results give us independent estimate of the refractive index for the compacted fused silica layer at an average of 1.484 which is 1.8 percent larger than that of the surrounding fused silica (1.457).

The refractive index increase can also be related to an increase in density for the layer using the LorenzLorent ${ }^{19}$ relation assuming that the molar refractivity remains nearly constant.

3) $\frac{1}{\rho_{1}}\left(\frac{\left(n_{1}\right)^{2}-1}{\frac{\left(n_{1}\right)^{2}}{3}+2}\right)=\frac{1}{\rho_{2}}\left(\frac{\left(n_{2}\right)^{2}-1}{\frac{\left(n_{2}\right)^{2}}{3}+2}\right)$

The compacted layer has a density estimated in the vicinity of $\rho_{2}=2.32 \mathrm{~g} / \mathrm{cc}$ which is 5.5 percent larger than that of normal fused silica $\rho_{1}=2.2 \mathrm{~g} / \mathrm{cc}$. Both the refractive index and the density increase of the compacted fused silica layer fall in the ranges established during experiments on fused silica under ultrahigh pressures ${ }^{19-22}$, Figure 12.

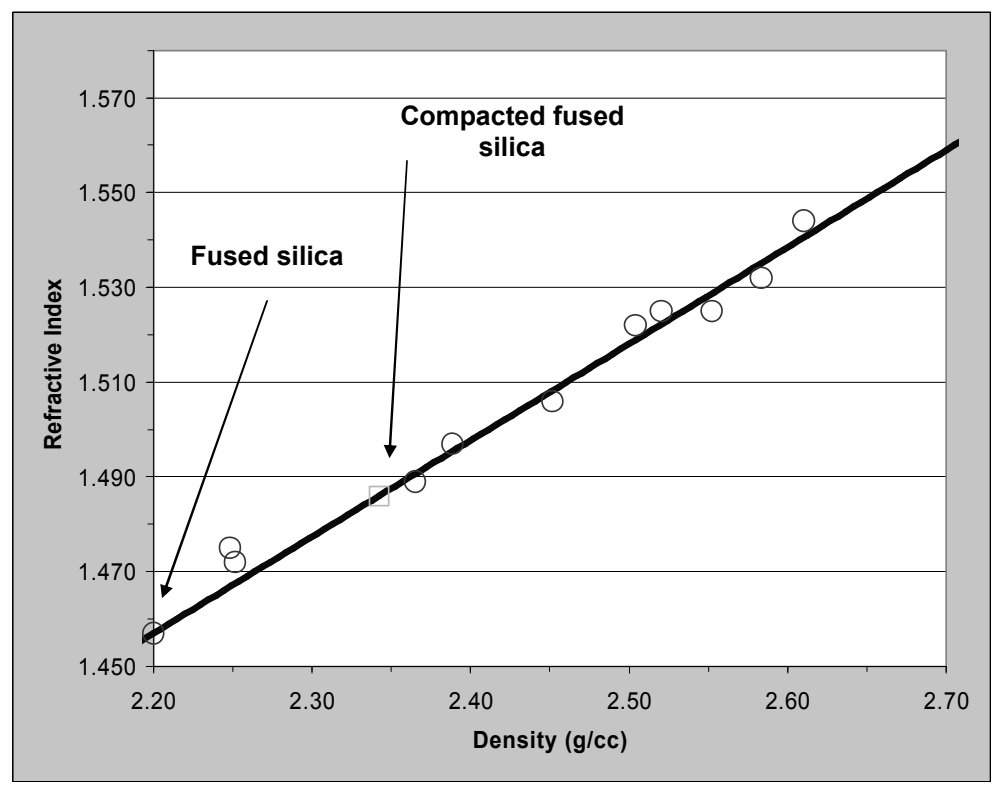

Data from H.M. Cohen and R. Roy,“Comments on Effects of Ultrahigh Pressures on Glass”, J.
Am. Ceram. Soc. 45[8] 398-99, 1962.

Figure 12: Comparison between the refractive index and density estimated for the compacted fused silica layer observed in a Knoop indentation and investigations conducted on fused silica at ultrahigh pressures. 


\section{SUMMARY AND CONCLUSIONS}

MRF techniques have been combined with laboratory analytical tools to develop a process that can be used to study the details of fractures and imperfections in optical materials. This three-dimensional MRF process involves a finishing process where AOIs on an optical surface can be cross-sectioned at specified intervals using the MRF and examined at each stage. Both individual fractures from engineered specimens and fracture networks from process related samples can be interrogated. The generation of three-dimensional constructs for the imperfections has offered the ability to resolve structural details of the fractures and fracture networks. The novel cross-sectioning technique and characterization routines have aided in the understanding the profile and shape of fractures as one moves into bulk material. It has also led to the identification and characterization of modified zones possessing different refractive indices and densities. For fused silica, we found that technique can provide a detailed picture of the lateral and radial cracking that occurs during various grinding and polishing processes. We have observed that radial fractures dominate an optic's subsurface damage character, particularly the last or most recent fractures generated from a grinding process. These fractures have a characteristic morphology peculiar to the process type and material removal conditions which results in a signature for the effects of the process used. Older radial fractures are reduced in depth and ultimately removed via lateral fracture generation. Lateral fractures are, therefore, responsible for the extent of material removal for a given process. This system of material removal and characterization has enabled us to gain an understanding of how optical fabrication processes interact with an optical surface and has led us to an understanding of how to optimize the optical fabrication process so that higher quality optics can be manufactured using the best known methods and material removal protocols. Through the study of engineered imperfections, we are able to observe the elastic nature of fused silica. Here, we found that fused silica can be elastically deformed between 45 and 50 percent during surface compression. It also has a unique attribute in that it can be compacted under load yielding a stable modified zone of finite dimensions. This modified material possesses a refractive index about 1.8 percent larger and a density about 5.5 percent larger than normal fused silica.

\section{REFERENCES}

1. F. Preston, "Structure of abraded surface glasses", Trans. Opt. Soc., 23(3), 141-162 (1922).

2. H. Karow, "Fabrication Methods for Precision Optics" John Wiley \& Sons (1993).

3. T. Kamimura, S. Akamatsu, H. Horibe, H. Shiba, S. Motokoshi, T. Sakamoto, T. Jitsuno, T. Okamoto, and K. Yoshida, "Enhancement of Subsurface-Damage Resistance by Removing Subsurface Damage in Fused Silica and Its Dependence on Wavelength", Japanese Journal of Applied Physics, 43, 9A/B, 1229-1231 (2004).

4. N. Brown, "Optical Fabrication", Lawrence Livermore National Laboratory Report, MISC 4476, August 1989.

5. T. Yoshino, Y. Kurata, Y. Terasaki, and K. Susa, "Mechanisms of polishing of $\mathrm{SiO}_{2}$ films by $\mathrm{CeO}_{2}$ particles", Journal of Non-Crystalline Solids, 283, 129-136 (2001).

6. D. W. Camp, M. R. Kozlowski, L. M. Sheehan, M. Nichols, M. Dovik, R. Raether, I. Thomas, "Subsurface damage and polishing compound effect at the 355-nm laser damage threshold of fused silica surfaces", Laser Induced Damage in Optical Materials, SPIE Proc., 3244, 356-364 (1997).

7. G. Beilby, "Aggregation and Flow of Solids", 1st Ed, London: Macmillan and Co. (1921).

8. J. A. Menapace, B Penetrante, D. Golini, A. Slomba, P. E. Miller, T Parham, M Nichols, and J. Peterson, "Combined Advanced Finishing and UV-Laser Conditioning for Producing UV-Damage-Resistant Fused Silica Optics", Laser Induced Damage in Optical Materials, SPIE Proc., 4679, 56-67 (2002).

9. J. A. Menapace, S. N. Dixit, Francois Y. Génin, and W. F. Brocious, "Magnetorheological Finishing for Imprinting Continuous Phase Plate Structure onto Optical Surfaces", Laser Induced Damage in Optical Materials, SPIE Proc., 5273, 220-230 (2003).

10. S. D. Jacobs, D. Golini, Y. Hsu, B.E. Puchebner, D. Strafford, W. I. Kordonski, I. V. Prokhorov, E. Fess, D. Pietrowski, and V. W. Kordonski," Magnetorheological finishing: a deterministic process for optics manufacturing", SPIE, 2576, 372-382 (1995). 
11. P. E. Miller, T. I. Suratwala, J. P. Menapace, L. L. Wong, P. J. Davis, and W. A. Steele, "The Distribution of Subsurface Damage in Fused Silica" Laser Induced Damage in Optical Materials, this proceedings, Boulder, Co (2005).

12. T. Suratwala, L. Wong, P. Miller, M. D. Feit, J. Menapace, R. Steele, P. Davis, D. Walmer, "Sub-surface mechanical damage distributions during grinding of fused silica", Journal of the American Ceramics Society, in submission.

13. G. F. Vander Voort and G. Lucas, "Microindentation Hardness Testing", Advanced Materials and Processes, September Ed. (1998).

14. J. A. Menapace, P. J. Davis, W. A. Steele, L. L. Wong, T. I. Suratwala, and P. E. Miller, "MRF Applications: Measurement of Process-dependent Subsurface Damage in Optical Materials using the MRF Wedge Technique", Laser Induced Damage in Optical Materials, this proceedings, Boulder, Co (2005).

15. Similar observations for Vickers indentations have been made by N. Gougeon, R. El Abdi, and M. Poulain, "Influence of the structural relaxation on the indentation behavior of the surface of silica optical fibers", World Conference on Non-destructive Testing, Montreal Canada (2004).

16. L. Harris, J. K. Beasley, and A. L. Loeb, "Reflection and Transmission of Radiation by Metal Films and the Influence of Nonabsorbing Backings", Journal of the Optical Society of America, 41, 9, 604-614, (1951).

17. T. Doi, K. Toyoda, and Y. Tanimura, "Effects of phase changes on reflection and their wavelength dependence in optical profilometry", Applies Optics, 36, 7157-7161 (1997)

18. A. Harasaki, J. Schmitt, and J.C. Wyant, "Offset of coherent envelop position due to phase change on reflection", Applied Optics, 40, 2102-2105 (2001).

19. H. M. Cohen and R. Roy, "Reply to Comments on Effects of Ultrahigh Pressure on Glass", Journal of the American Ceramics Society, 45, 398-399 (1962).

20. J. D. Mackenzie, "High-Pressure Effects on Oxide Glasses: 1, Densification in Rigid State", Journal of the American Ceramic Society, 46, 461-470 (1963).

21 F. M. Ernsberger, "Role of Densification in Deformation of Glass under Point Loading", Journal of the American Ceramic Society, 51, 545-547 (1968).

22. H. M. Cohen and R. Roy, "Effects of Ultrahigh Pressure on Glass", Journal of the American Ceramic Society, 44, 523-524 (1962). 